\title{
VARIACIONES EN LAS CONCENTRACIONES SÉRICAS DE CALCIO, FÓSFORO Y POTASIO EN PERROS CON ENFERMEDAD RENAL CRÓNICA EN DIFERENTES ESTADIOS: UN ESTUDIO PRELIMINAR EN PARAGUAY
}

\author{
VARIATIONS IN CALCIUM, PHOSPHORUS AND POTASSIUM SERUM CONCENTRATIONS IN DOGS WITH \\ CHRONIC KIDNEY DISEASE AT DIFFERENT STAGES: A PRELIMINARY STUDY IN PARAGUAY
}

\section{Pedrozo Prieto R, Domel B.}

Departamento de Patología y Clínica, División Patología Clínica, Facultad de Ciencias Veterinarias, Universidad Nacional de Asunción - San Lorenzo - Paraguay

RESUMEN: Con el objetivo de evaluar las variaciones de las concentraciones séricas de calcio (Ca), fósforo (P) y potasio (K) en perros con enfermedad renal crónica (ERC), 61 perros con diagnóstico clínico de ERC, fueron clasificados en 5 estadios de acuerdo al sistema de la International Renal Interest Society (IRIS). Los valores de urea, creatinina, calcio, fósforo y potasio fueron determinados por espectrofotometría y los resultados analizados con los estadísticos Kruskal Wallis y Correlación de Spearman. Como resultado, no existieron diferencias al comparar los valores de calcio en los 5 estadios de la ERC ( $p=0,19)$, sin embargo la frecuencia de hiperfosfatemia aumentó con la severidad de ERC. Se observaron diferencias al comparar las concentraciones séricas de fósforo $(\mathrm{p}<0,0001)$ habiendo un incremento importante solo en los estadios 3 y 4 . Por otro lado, existió diferencia significativa entre la media de los valores de potasio en el estadio 4 con relación a las otras fases $(\mathrm{p}<0,0001)$. Las concentraciones séricas de fósforo y potasio fueron correlacionadas significativa y positivamente, con la concentración sérica de urea y creatinina $(\mathrm{p}<0,0001$ y 0,003$)$. Sin embargo el calcio tuvo una asociación muy débil con urea y débil con creatinina, con correlaciones positivas y no significativas ( $\mathrm{p}=0,87$ y 0,09 ).

Palabras clave: calcio, fósforo, potasio, perros, enfermedad renal crónica.

\begin{abstract}
In order to evaluate changes in serum calcium (Ca), phosphorus (P) and potassium (K) in dogs with chronic kidney disease (CKD), 61 dogs with clinical diagnosis of CKD were classified into 5 stages according to the system of the International Renal Interest Society (IRIS). The values of urea, creatinine, calcium, phosphorus and potassium were determined by spectrophotometry and the results analyzed with statistical Kruskal Wallis and Spearman correlation. As a result, there were no differences when comparing the values of calcium in the 5 stages of CKD ( $p=0.19)$, but the frequency of hyperphosphatemia increased with the severity of CKD. Differences were observed when comparing the serum concentrations of phosphorus $(\mathrm{p}<0.0001)$ having a significant increase only in stages 3 and 4 . On the other hand, there was significant difference between the mean values of potassium in the stage 4 relative to the other phases $(p<0.0001)$. Serum concentrations of phosphorus and potassium were significantly and positively correlated with serum urea and creatinine $(\mathrm{p}<0.0001$ and 0.003$)$. However calcium had a weak association with urea and weaker with creatinine, with positive correlations and not significant $(\mathrm{p}=0.87$ and 0.09$)$.
\end{abstract}

Keywords: calcium, phosphorus, potassium, dogs, chronic renal disease. 


\section{INTRODUCCIÓN}

Los riñones cumplen un papel crucial en la homeostasis, por ser el principal órgano de excreción del exceso de productos de desechos nitrogenados, controlando la concentración en el plasma de muchos electrolitos, dentro de unos límites muy estrechos. Estos incluyen iones de sodio, potasio, cloro, fósforo y magnesio. Los riñones son capaces de excretar o reabsorber cantidades variables de cada electrolito en respuesta a las necesidades del cuerpo (1).

Las nefropatías crónicas son las enfermedades renales más frecuentes en la clínica de pequeños animales y una de las principales causas de mortalidad en pacientes de edad avanzada (2). Las lesiones renales conducen no sólo a las complicaciones derivadas de la acumulación de toxinas urémicas y desequilibrios electrolíticos, sino también a la hipertensión arterial sistémica, empeorando el estado clínico y comprometiendo otros órganos como el corazón (3).

La enfermedad renal crónica (ERC) puede clasificarse en diferentes fases a lo largo de un espectro continuo de enfermedad progresiva. La utilidad de esta clasificación radica en poder facilitar la aplicación de unas directrices clínicas adecuadas tanto para el diagnóstico, pronóstico como tratamiento (2). Tanto en la medicina humana y veterinaria, el diagnóstico y la categorización de la enfermedad renal puede ser difícil. La medición de la tasa de filtración glomerular se considera el "estándar de oro" para la evaluación de la función renal, no obstante los métodos para su evaluación pueden ser técnicamente difíciles y poco prácticos. Los principales parámetros que se utilizan para diagnosticar la enfermedad renal aguda y crónica incluyen las concentraciones circulantes de creatinina y urea, y la gravedad específica de la orina (4).

Las concentraciones de urea y creatinina en plasma y suero son rutinariamente analizadas como marcadores indirectos de la tasa de filtración glomerular y se ha reportado que están altamente correlacionadas $(5,6)$. La medición simultánea de ambas es de valor diagnóstico limitado sobre el análisis de una sola variable. La creatinina es el analito preferido, al verse afectado por un menor número de factores extrarrenales de variación (6).
La concentración de creatinina circulante es el marcador de función renal más ampliamente utilizado en medicina humana y veterinaria. El sistema International Renal Interest Society - (IRIS) usa como indicador la concentración de creatinina para categorizar perros y gatos con ERC, y los estadios de IRIS han sido fuertemente asociados con el tiempo de supervivencia (4).

La evidencia disponible en seres humanos y gatos con enfermedad renal crónica espontánea, y en perros con ERC experimental, indica que la valoración de alteraciones del metabolismo de calcio y fósforo es una parte importante de la evaluación de laboratorio en estos pacientes, debido a sus efectos negativos sobre la función renal y la supervivencia. En los perros con ERC experimental, la hiperfosfatemia se ha asociado con una progresión más rápida de la ERC y la disminución de la supervivencia (7).

La hiperkalemia parece ser un efecto secundario común de la inhibición del Sistema renina-angiotensina-aldosterona (RAAS) en perros con enfermedad renal. Los perros con las concentraciones de potasio en suero mayor a 6 mmol/L deben ser monitoreados de cerca (8).

El objetivo del presente trabajo fue evaluar las variaciones existentes en las concentraciones séricas de calcio total, fósforo inorgánico y potasio en perros clasificados en diferentes estadios de enfermedad renal crónica.

\section{MATERIALES Y MÉTODOS}

El estudio fue realizado en el Hospital Veterinario "Prof. Dr. Vicente Núñez" del Departamento de Patología y Clínica de la Facultad de Ciencias Veterinarias de la Universidad Nacional de Asunción (San Lorenzo, Paraguay). Fueron seleccionados 61 caninos con anamnesis, síntomas clínicos y resultados del urianálisis compatibles con afección renal, de por lo menos 2 semanas de duración, para determinar cronicidad, que consultaron en el referido Hospital durante el año 2013. De cada perro se obtuvo $3 \mathrm{~mL}$ de sangre, mediante punción de la vena cefálica, depositada en tubos, que fueron derivadas al laboratorio.

Las muestras de suero fueron procesadas en el Laboratorio de la División de Patología Clínica 
dependiente del mismo Departamento, determinándose la concentración de los siguientes analitos: urea, por el método de ureasa Berthelot modificado a $37^{\circ} \mathrm{C}(600 \mathrm{~nm})$; creatinina, por el método de Jaffe sin desproteinización a $37^{\circ} \mathrm{C}(505$ $\mathrm{nm}$ ); calcio total (calcio), por el método oCresoftaleína-complexona a $25^{\circ} \mathrm{C}(560 \mathrm{~nm})$; fósforo inorgánico (fósforo), por el método Fosfomolibdato a $25^{\circ} \mathrm{C}(340 \mathrm{~nm})$ y potasio, por el método Tetrafenilboron a $25^{\circ} \mathrm{C}(600 \mathrm{~nm})(9)$. Se utilizaron kits reactivos Human (Alemania) y fotómetro Biosystems BTS 350 (España).

Con el fin de correlacionar la gravedad de la lesión renal con la concentración sérica de calcio, fósforo y potasio, los perros fueron divididos en grupos de acuerdo al sistema de clasificación de la IRIS para la ERC (10). El primer grupo (E0), estadio en riesgo a desarrollar ERC por diversos factores (exposición a drogas nefrotóxicas, serología positiva a Leishmania infantum, edad avanzada), estuvo compuesto por 15 perros, el segundo grupo (E1) estuvo compuesto por 25 perros incluidos en la estadio 1 de la ERC, es decir, con la pérdida de la capacidad de concentrar la orina, proteinuria persistente, valores de creatinina $<1,4 \mathrm{mg} / \mathrm{dL}$. El tercer grupo (E2) consistió en 2 perros que clasificaron en el estadio 2 de ERC (creatinina sérica entre 1,4 y $2,0 \mathrm{mg} / \mathrm{dL}$, sin signos clínicos o signos leves de enfermedad renal); en el cuarto grupo (E3) 4 perros se incluyeron en el estadio 3 (creatinina sérica entre 2,1 y 5,0 mg/dL, con signos sistémicos de ERC) y 15 perros se incluyeron en el estadio 4 (E4) de la enfermedad renal crónica, con signos clínicos sistémicos y crisis urémicas.

Para cada analito se obtuvieron los valores promedios y desviación estándar (DE) de los 5 grupos constituidos, los que fueron comparados utilizando las pruebas de ANOVA y Kruskal Wallis cuando no se cumplió el supuesto de homogeneidad de varianzas (11); y para las comparaciones de las medias fueron empleadas pruebas estadísticas de Tukey y Trat Ranks. El test de correlación de Spearman fue aplicado para identificar correlaciones entre las variables estudiadas; utilizando para el análisis el paquete estadístico InfoStat (versión estudiantil) (12). Se consideró la diferencia estadísticamente significativa cuando $\mathrm{p}<0,05$.

\section{RESULTADOS Y DISCUSIÓN}

El grupo de perros estudiados, con diagnóstico clínico de alteración renal crónica incluyó a 61 perros ( 29 hembras y 32 machos). Sus edades estuvieron comprendidas entre 2 y 14 años (promedio $8 \pm 3,1$ años de DE). Sus razas comprendían 19 mestizos, 8 Caniches, 7 Cocker Spaniels, 5 Teckel, 5 Pastor Alemán, 4 Boxers, 3 Dálmatas, 3 Pitbull, 2 Rottweiler, 1 Chow Chow, 1 Fox Terrier, 1 Gran Danés, 1 Sharpei y 1 Weimaraner.

La población se clasificó en 5 estadios, una fase de riesgo (E0) y 4 estadios de enfermedad renal crónica. Los estadios con mayor número de individuos fueron el $0,1 \mathrm{y} 4$.

Los intervalos de referencia de las distintas variables difieren en la bibliografía, se tuvo en cuenta los de Meyer y Harvey 2007, Kaneko 2008 y Wittwer $2012(13,14,15)$. En la Tabla 1 se presentan los resultados agrupados según estadio de ERC.

La media de las concentraciones séricas de calcio en los estadios 0 y 1 , se encontró dentro del intervalo de referencia, en el estadio 2 por debajo,

Tabla 1. Concentración sérica de urea, creatinina, calcio, fósforo y potasio en caninos en diferentes estadios de enfermedad renal crónica $(\mathrm{n}=61)$.

\begin{tabular}{|c|c|c|c|c|c|}
\hline $\begin{array}{l}\text { Estadio ERC } \\
\text { (IRIS)* }^{*} \\
\text { (Intervalo de } \\
\text { Referencia) }\end{array}$ & $\begin{array}{l}\text { Urea } \\
(\mathrm{mg} / \mathrm{dL}) \\
(16-40) \\
\text { Media } \pm \text { DE }\end{array}$ & $\begin{array}{l}\text { Creatinina } \\
(\mathrm{mg} / \mathrm{dL}) \\
(0,5-1,4) \\
\text { Media } \pm \mathrm{DE}\end{array}$ & $\begin{array}{l}\text { Calcio } \\
(\mathrm{mg} / \mathrm{dL}) \\
(9-11) \\
\text { Media } \pm \mathrm{DE}\end{array}$ & $\begin{array}{l}\text { Fósforo } \\
\text { (mg/dL) } \\
(2,6-6,2) \\
\text { Media } \pm \text { DE }\end{array}$ & $\begin{array}{l}\text { Potasio } \\
(\mathrm{mmol} / \mathrm{L}) \\
(3,7-5,7) \\
\text { Media } \pm \mathrm{DE}\end{array}$ \\
\hline E0 $(n=15)$ & $30 \pm 10,5^{a}$ & $0,6 \pm 0,2$ a & $10,7 \pm 2,0$ & $5,4 \pm 1,3^{a}$ & $4,9 \pm 1,4^{a}$ \\
\hline $\mathrm{E} 1(\mathrm{n}=25)$ & $62 \pm 21,2^{a}$ & $0,9 \pm 0,3 \mathrm{~b}$ & $10,1 \pm 1,4$ & $5,7 \pm 1,1^{\mathrm{ab}}$ & $5,4 \pm 1,7 a$ \\
\hline $\mathrm{E} 2(\mathrm{n}=2)$ & $99 \pm 49,5^{\mathrm{a}}$ & $1,7 \pm 0,2 \mathrm{bc}$ & $8,5 \pm 2,4$ & $5,6 \pm 2,6^{a b}$ & $4,2 \pm 0,1^{\mathrm{a}}$ \\
\hline E3 $(n=4)$ & $175 \pm 57,5^{a}$ & $2,4 \pm 0,3 \mathrm{c}$ & $11,3 \pm 6,3$ & $14,1 \pm 9,2^{b c}$ & $5,5 \pm 2,6^{a}$ \\
\hline$E 4(n=15)$ & $432 \pm 229,1^{b}$ & $23,7 \pm 15,2 \mathrm{c}$ & $13,6 \pm 6,1$ & $22,6 \pm 7,7 c$ & $19,9 \pm 14,0^{b}$ \\
\hline ANOVA & $\begin{array}{l}F=29,70 \\
p=<0,0001\end{array}$ & $\begin{array}{l}H=46,47 \\
p=<0,0001\end{array}$ & $\begin{array}{l}H=6,10 \\
p=0,19\end{array}$ & $\begin{array}{l}H=35,76 \\
p=<0,0001\end{array}$ & $\begin{array}{l}F=12,0 \\
p=<0,0001\end{array}$ \\
\hline
\end{tabular}

*ERC: Enfermedad renal crónica IR: Intervalo de referencia

** a b c Letras distintas indican diferencias entre estadios $(\mathrm{p}<0,05)$ 
considerando que en este grupo hubieron solo dos individuos, pero por encima del rango de referencia en los estadios 3 y 4 (Tabla 1 ).

No existieron diferencias significativas al comparar los valores de calcio total en los 5 estadios $(p=0,19)$ (Tabla 1). Estos resultados coinciden en parte con Cordatellas et al 2010, donde no se detectaron diferencias entre el grupo control y de perros enfermos (7). Sin embargo, en el mismo trabajo existieron discrepancias cuando se midieron calcio total y calcio iónico. El calcio total sobreestima la prevalencia de hipercalcemia y subestima la prevalencia de hipocalcemia (7).

Cuando los valores individuales fueron analizados (Tabla 2), existieron pacientes con hipo, normo e hipercalcemia en los estadios 0 y 1 , individuos con hipo y normocalcemia en el estadio 2 , caninos con hipo e hipercalcemia en el estadio 3, pacientes con hipo y mayor parte hipercalcemia $(66,7 \%)$ en el estadio 4. Estos resultados no coinciden con Cordatellas et al 2009, donde la hipercalcemia fue reportada en un solo perro del estadio 3 y no hubo pacientes con hipocalcemia (16).

En la alteración renal crónica hay una pérdida de la capacidad de reabsorción de calcio, con consecuente hipocalcemia, tal como lo reportan varios autores $(1,13,15)$, la que al ser crónica, estimula la paratiroides a movilizar calcio óseo para mantener la homeostasis, culminando en el hiperparatiroidismo secundario renal (1). Por otro lado, los cuadros de hipoalbuminemia asociados a albuminuria pueden cursar con hipocalcemia (15). La hipocalcemia puede ser debida además, a la precipitación del fosfato cálcico en la sangre, por resistencia del esqueleto a los efectos de la paratohormona (PTH) (1). La hipercalcemia ocasionalmente ocurre en perros con enfermedad renal, pudiendo también ser causa de enfermedad renal (13), esto puede ser debido al aumento de los complejos de calcio ligado a fosfatos, oxalatos y otros aniones en sangre (1). Lo señalado anteriormente explica la presencia de pacientes con hipo y normocalcemia en los estadios 0,1 y 2 , así como la mayor cantidad de caninos con hipercalcemia en los estadios más avanzados 3 y 4 .

Evaluando las concentraciones séricas de fósforo, la media se encontró dentro del intervalo de referencia en los estadios 0,1 y 2 , y por encima en los estadios 3 y 4, existiendo diferencias significativas $(\mathrm{p}<0,0001)$ al comparar las medias de fósforo en los diferentes estadios de ERC, aumentando la misma con la progresión de la alteración renal (Tabla 1).

Al evaluar los valores individuales de fósforo sérico, se ve que las proporciones de caninos con normofosfatemia disminuyen del estadio 0 al 3. Sin embargo, los pacientes con hiperfosfatemia van aumentando con la progresión de la ERC, siendo de 75 y $100 \%$ en los estadios más avanzados 3 y 4 , respectivamente. Valores muy similares son reportados en algunos estudios $(7,13)$.

En pacientes con fallo renal, la alteración que aparece con mayor frecuencia es la hiperfosfatemia, que se desarrolla como consecuencia directa de la disminución del índice de filtración glomerular (IFG) $(1,13,16)$. La hiperfosfatemia promueve lesiones renales progresivas y ha sido relacionada al aumento de mortalidad en humanos y perros con ERC (16). Esta relación puede explicarse por dos mecanismos, primero, la retención de fósforo asociada con el desarrollo y progresión de un hiperparatiroidismo secundario $(1,15,16)$, que exacerba la progresión de ERC debido a sus efectos en huesos, riñones, cerebro y otros órganos. Secundariamente, la retención de fósforo promueve la progresión de ERC por la precipitación de calcio en el intersticio renal como fosfato de calcio, resultando en fibrosis intersticial y

Tabla 2. Análisis de valores individuales de calcemia, fosfatemia y kalemia en cuanto a cantidad (n) y proporción (\%) de pacientes.

\begin{tabular}{l|ccc|cc|ccc}
\hline Estadio & \multicolumn{3}{|c|}{ Calcemia n (\%) } & \multicolumn{2}{c|}{ Fosfatemia n (\%) } & \multicolumn{3}{c}{ Kalemia n (\%) } \\
ERC (n) & Hipo & Normo & Hiper & Normo & Hiper & Hipo & Normo & Hiper \\
\hline E0 (15) & $1(6,6)$ & $7(46,7)$ & $7(46,7)$ & $11(73,3)$ & $4(26,7)$ & ------ & $12(80)$ & $3(20)$ \\
E1 (25) & $5(20)$ & $14(56)$ & $6(24)$ & $16(64)$ & $9(36)$ & $3(12)$ & $16(64)$ & $6(24)$ \\
E2 (2) & $1(50)$ & $1(50)$ & ------ & $1(50)$ & $1(50)$ & ----- & $2(100)$ & ----- \\
E3 (4) & $2(50)$ & ------ & $2(50)$ & $1(25)$ & $3(75)$ & $2(50)$ & ------ & $2(50)$ \\
E4 (15) & $4(26,7)$ & $1(6,6)$ & $10(66,7)$ & ------ & $15(100)$ & ----- & $4(26,7)$ & $11(73,3)$ \\
\hline
\end{tabular}


atrofia tubular (16). De acuerdo con los resultados de Cordatellas et al 2010, que estudiaron la homeostasis de calcio y fósforo, el fosfato sérico predice con bastante precisión la existencia de hiperparatiroidismo renal (7).

Las fluctuaciones en la proporción calcio : fósforo se visualizan en la Figura 1 (A). La proporción óptima Ca:P fluctúa entre 2:1 y 1:1 (17). Se evidenció alteración en los estadios 3 y 4 con proporciones 0,8:1 y $0,6: 1$.
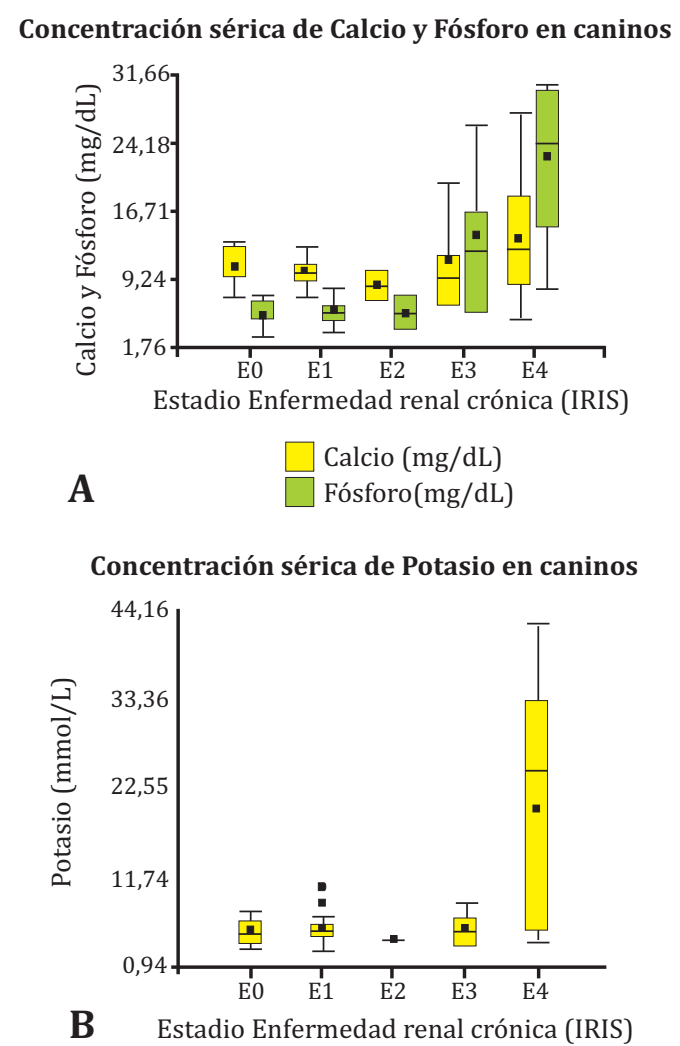

Figura 1. (A) Concentración sérica de calcio, fósforo y (B) Concentración sérica de potasio en caninos clasificados en distintos estadios de ERC. Cada box-plot ilustra la media, mediana y cuartiles.

La media de las concentraciones séricas de potasio se encontró dentro del intervalo de referencia en los estadios $0,1,2$ y 3 , y por encima en el estadio 4, con diferencia estadísticamente significativa $(\mathrm{p}<0,0001)$ (Tabla 1$)$.
La mayoría de los individuos presentó normokalemia en los estadios 0, 1 y 2. El 50\% de los caninos presentó hipo e hiperkalemia en el estadio 3, mientras que el 73,3\% de los pacientes presentó hiperkalemia en el estadio 4(Tabla 2). Por otro lado, a llamado la atención el amplio rango de valores de potasio en el estadio 4, como puede verse en la Figura 1 (B).

En los perros, el potasio del plasma suele permanecer normal durante las fases poliúricas del fallo renal y aumentan durante la oliguria o la anuria de la ERC (1). Animales con ERC terminal presentan hiperkalemia $(1,15)$ lo que coincide con esta investigación. Segev et al 2010 reportaron que la hiperkalemia es una complicación potencial de la ERC, teniendo el $47 \%$ de la población uno o más episodios de hiperkalemia, en algunos casos grave (18).

Las concentraciones séricas de urea y creatinina en este trabajo, fueron correlacionadas significativa y positivamente $(\mathrm{p}<0,0001)$, siendo rho $=0,77$ (Tabla 3), presentando una asociación fuerte, debido a que es mayor a 0,7 según lo describe Martínez et al 2008 (11). Estos resultados coinciden con Medaille et al 2004, que encontraron una asociación altamente significativa $(r=0,8)(5)$. Sin embargo Braun et al 2003 en una revisión, afirmaron que en perros con ERC espontánea o inducida quirúrgicamente, la correlación entre concentraciones plasmáticas de urea y creatinina fue reportada como alta o baja (19).

Las concentraciones séricas de fósforo y potasio fueron correlacionadas significativa $\mathrm{y}$ positivamente rho $=0,66$ y 0,38 , con la concentración sérica de urea $(\mathrm{p}<0,0001$ y 0,003$)$, con asociaciones moderadas. Sin embargo, el calcio tuvo una asociación muy débil con la urea rho $=0,02$; con correlación positiva y no significativa $(\mathrm{p}=0,87)$ (Tabla $3)$.

Tabla 3. Correlación de Spearman entre las variables analizadas.

\begin{tabular}{lllcr}
\hline Variable(1) & Variable(2) & $\mathrm{n}$ & Spearman & \multicolumn{1}{c}{ p-valor } \\
\hline Urea (mg/dL) & Creatinina (mg/dL) & 61 & 0,77 & $<0,0001$ \\
Urea (mg/dL) & Calcio (mg/dL) & 61 & 0,02 & 0,8712 \\
Urea (mg/dL) & Fósforo (mg/dL) & 61 & 0,66 & $<0,0001$ \\
Urea (mg/dL) & Potasio (mmol/L) & 61 & 0,38 & 0,0027 \\
& & & & \\
Creatinina (mg/dL) & Urea (mg/dL) & 61 & 0,77 & $<0,0001$ \\
Creatinina (mg/dL) & Calcio (mg/dL) & 61 & 0,22 & 0,0886 \\
Creatinina (mg/dL) & Fósforo(mg/dL) & 61 & 0,71 & $<0,0001$ \\
Creatinina (mg/dL) & Potasio (mmol/L) & 61 & 0,38 & 0,0025 \\
\hline
\end{tabular}


Las concentraciones séricas de fósforo y potasio fueron correlacionadas significativa $\mathrm{y}$ positivamente, rho $=0,71$ y 0,38 , con la concentración sérica de creatinina ( $\mathrm{p}<0,0001$ y 0,003$)$; con asociaciones fuerte y moderada, respectivamente. El calcio tuvo una asociación débil con creatinina rho $=0,22$; con correlación positiva y no significativa $(\mathrm{p}=0,09)$ (Tabla 3$)$.

Los resultados coinciden parcialmente con lo reportado por Cordatellas et al 2009, y son esperados, en el caso fósforo sérico, éste aumenta con la severidad de ERC (16). Sin embargo, estos autores comunicaron una relación más fuerte entre fósforo y urea séricos que entre fósforo y creatinina séricos (16), siendo opuestos a los hallazgos en esta investigación.

\section{CONCLUSIÓN}

No existieron diferencias al comparar los valores de calcio en los 5 estadios de la enfermedad renal crónica en caninos.

La presencia de hiperfosfatemia aumentó con la severidad de ERC. Existieron diferencias al comparar las concentraciones séricas de fósforo, habiendo un incremento importante en los estadios más avanzados 3 y 4 .

Existió diferencia significativa entre la media de los valores de potasio en el estadio 4 con relación a las otras fases, siendo algunos valores muy elevados y el rango amplio en la última fase de ERC.

Las concentraciones séricas de fósforo y potasio fueron correlacionadas significativa y positivamente, con la concentración sérica de urea y creatinina. Sin embargo el calcio tuvo una asociación muy débil con urea y débil con creatinina.

\section{BIBLIOGRAFÍA}

1. Williers E, Backwood L. Manual de diagnóstico de laboratorio en pequeños animales. $2^{\mathrm{a}}$ ed. Barcelona: Lexus; 2012.

2. Suárez ML. Manejo de la Enfermedad renal crónica. RECVET (revista en internet) 2007 enero-abril. (acceso 18 de mayo de 2015 ); 1 ( 4 ). D i s p o n i b l e e n : http://www.veterinaria.org/revistas/recvet/n01a0407.htlm.

3. Braga E, Carvalho J, Rosa F, Tivelli P, Araújo A, Almeida B et al. Hypertension and its correlation with renal lesions in dogs with leishmaniosis. Braz. J. Vet. Parasitol. 2015; 24 (1): 45-51.
4. Cobrin A, Blois S, Kruth S, Abrams-Ogg A, Dewey C. Biobarmerks in the assessment of acute and chronic kidney diseases in the dog and cat. J. Small Ani. Pract. 2013; 54: 647-655.

5. Medaille C, Trumel C, Concordet D, Vergez F, Braun JP. Comparison of plasma/serum urea and creatinine concentrations in the dog: a 5-year retrospective study in a commercial Veterinary Clinical Pathology Laboratory. J. Vet. Med. 2004; 51: 15.

6. Concordet D, Vergez F, Trumel C, Diquelou A, Lanore D, Le Garreres A et al. A multicentric retrospective study of serum/plasma urea and creatinine concentrations in dogs using univariate and multivariate decision rules to evaluate diagnostic efficiency. Vet. Clin. Pathol. 2008; 1-5.

7. Cordatellas 0 , Fernández-del Palacio M, Talavera J, Bayón A. Calcium and phosphorus homeostasis in dogs with spontaneous chronic kidney disease at different stages of severity. J. Vet. Med. $2010 ; 24: 73-79$.

8. Brown S, Elliott J, Francey T, Polzin D, Vaden S. Consensus recommendations for standard therapy of glomerular disease in dogs. J. Vet. Intern. Med. 2013; 27: 27-43.

9. Burtis C, Bruns D. Tietz Fundamentals of clinical chemistry and molecular diagnostics. 7a ed. Philadelphia: Saunders; 2014.

10. International Renal Interesty Society - IRIS. IRIS staging of CKD (Página de inicio en Internet). 2013. (acceso 23 de abril de 2015). Disponible en: http://www.iris-kidney.com

11. Martínez-González M, Sánchez-Villegas A, Faulín J. Bioestadística amigable. 2a ed. Navarra: Díaz de Santos; 2008.

12. Sofware estadístico InfoStat. Facultad de Ciencias Agropecuarias. Universidad Nacional de Córdoba, Argentina; 2014 . (24 de abril de 2015) Disponible en: http://www.fca.proed.unc.edu.ar/file.php./68/Tutorial_soft.pdf

13. Meyer D, Harvey J. Medicina laboratorial veterinaria: interpretación y diagnósis. 3a ed. Barcelona: Multimédica; 2007.

14. Kaneko J, Harvey J, Bruss M. Clinical biochemistry of domestic animals. 6a ed. San Diego: Academic Press; 2008.

15. Wittwer F. Manual de patología clínica veterinaria. 2a ed. Valdivia: América; 2012.

16. Cordatellas O, Fernández-del Palacio M, Talavera J, Bayón A. Serum phosphorus concentrations in dogs with leishmaniosis at different stages of chronic kidney disease. Vet. Rec. 2009; 164: $487-490$.

17. MacDonald P, Edwards R, Greenhalg. Nutrición animal. 3a ed. Zaragoza: Acribia; 1988.

18. Segev G, Fascetti AJ, Weeth LP. Correction of hyperkalemia in dogs with chronic kidney disease consuming commercial renal therapeutic diets by a potassium-reduced home-prepared diet. J. Vet. Intern. Med. 2010; 24 (3): 546 - 550.

19. Braun JP, Lefebvre H, Watson A. Creatinine in the dog: a review. Vet. Clin. Path. 2003; 32 (4): 162 - 179. 